\title{
Microscopic nuclear form factor for the pygmy dipole resonance
}

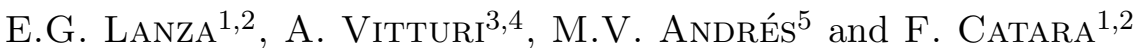

${ }^{1}$ INFN, Sezione di Catania, Catania, Italy

${ }^{2}$ Dipartimento di Fisica e Astronomia, Università di Catania, Italy

${ }^{3}$ Dipartimento di Fisica e Astronomia, Università di Padova, Italy

${ }^{4}$ INFN, Sezione di Padova, Padova, Italy

${ }^{5}$ Departamento de FAMN, Universidad de Sevilla, Spain

\begin{abstract}
Pygmy dipole resonance can be populated via isoscalar probes due to the strong mixing of isoscalar and isovector components. The physical quantities are usually extracted by the use of reaction theory calculations. There, the most important ingredient is the form factor which contains all the relevant properties of the states we are dealing with. We show that the use of microscopic form factor is required for a better analysis of experimental results.
\end{abstract}

\section{Introduction}

Among the most interesting new phenomena related to the nuclei with neutron excess, the low lying dipole states [1-3], also known as Pygmy Dipole Resonance (PDR), acquired a special role also for its connections with other branch of physics. The PDR states are clearly connected to the neutron skin of atomic nuclei [4]. Its isovector E1 strength is related to the isovector parameters in the equation of state of nuclear matter [5]. Furthermore, it is connected to the reaction rates in the astrophysical r-process which is responsible of the formation of the elements heavier than iron [6]. 

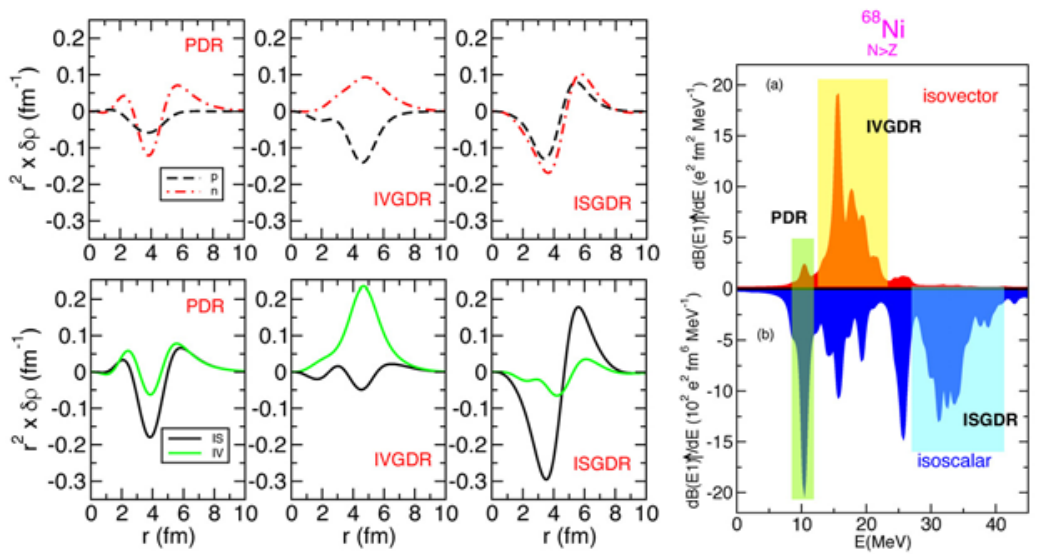

Figure 1: (Color online) RPA transition densities for three different dipole states for the nucleus ${ }^{68} \mathrm{Ni}$. In the frame at the right, the RPA isovector (upper part, red) and isoscalar (lower part, blue) response for the same nucleus (see text).

PDR states are better described within microscopic self consistent manybody approaches or on the relativistic meson-exchange Lagrangian [1]. All of these calculations show similar behaviors for the transition densities: the neutron and proton ones are in phase inside the nucleus while at the surface only the neutron part contributes significantly. This feature generates a strong mixing of isospin character and therefore even an isoscalar probe can be used to explore these low lying dipole states.

From the experimental point of view, these modes have been investigated using several methods on stable as well as on nuclei far from the stability line. The first evidence in unstable nuclei was found with a relativistic Coulomb excitation at GSI for the ${ }^{130,134} \mathrm{Sn}$ [7] and later on also for the ${ }^{68} \mathrm{Ni}$ [8]. Systematic studies on stable nuclei with the method of nuclear resonance fluorescence (NRF) have also been carried out on different mass regions [2]. Recently, the investigation of the nature of the PDR has been pursued via the use of isoscalar probes, namely via $\left(\alpha, \alpha^{\prime} \gamma\right)[2,9-12]$ or $\left({ }^{17} \mathrm{O},{ }^{17} \mathrm{O}^{\prime} \gamma\right)[3,13-15]$. Most of the physical information obtained by using isoscalar probes depend on how well the nuclear properties of the state under investigation are described within the radial form factors. Here, we present a comparison among different ways to calculate the radial nuclear form factor. Full details can be found in ref. [16].

\section{Transition densities and nuclear form factors}

Inelastic nuclear excitation is a useful method to extract information about nuclear structure. This is often done using different approaches 
like DWBA or a more complete description like the coupled channel models or their semiclassical versions especially developed for the studies of heavy ion collisions. The construction of the radial nuclear form factor is very important in all these approaches because it includes all the relevant information about the properties of the state under consideration. An extremely useful way to build up the form factors is through the well-known double folding procedure [17] which requires the knowledge of the transition density of the state under study. The transition densities can be calculated in a macroscopic or microscopic way according to the various models describing the different excitation modes.

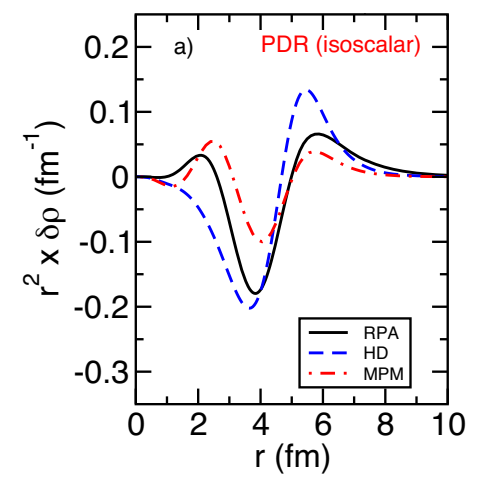

Figure 2: (Color online) Comparison among macroscopic isoscalar transition densities with the RPA based microscopic one (see text).
Microscopic transition densities can be obtained from several many body models calculations. As an example we have performed Hartree-Fock (HF) plus Random Phase Approximation (RPA) calculation with a SGII interaction [18] for the nucleus ${ }^{68} \mathrm{Ni}$. In the right part of fig. 1 the isovector (upper part, red) and isoscalar (lower part, blue) dipole response are shown with the three region of interest: the isovector giant dipole resonance (IVGDR) in the isovector part, the pure isoscalar giant dipole resonance at very high energy and the peak corresponding to the PDR centered at around $10 \mathrm{MeV}$. In the six frames on the left, the transition densities for the three states: the neutron (dot-dashed, red) and proton (dashed, black) transition densities in the upper part and the isovector (green) and isoscalar (black) one in the lower one. The IVGDR transition densities exhibit the well known behaviour with protons and neutrons oscillating out of phase giving rise to a strong isovector character of the mode; for the ISGDR, the protons and neutrons are oscillating in phase producing an isoscalar transition density with a node at the nuclear surface, typical of the compressional modes. For the PDR, neutrons and protons are in phase inside the nucleus while only the neutrons give a contribution at the surface. As a consequence, the isoscalar and isovector transition densities have the same strength at the surface which is the region of the nucleus explored in the nuclear reactions used to investigate the PDR states. These important features should be taken into account in the construction of the nuclear form factors. 


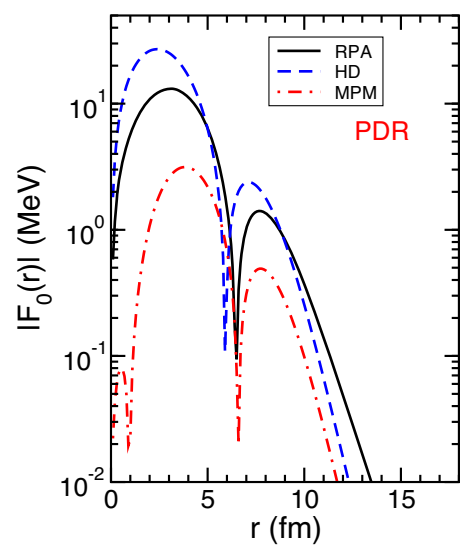

Figure 3: (Color online) Comparison among nuclear radial form factors calculated with the transition densities of fig.2 (see text).

compressional modes.

There is another way to calculate a transition density based on the common picture that one has in mind about the nature of the PDR mode. It is a widespread belief, in the literature, that the low lying dipole states are generate by an out-of-phase oscillation of the neutrons on the nuclear surface against a proton-neutron core. Assuming this picture, we have calculated the neutron and proton transition densities and the corresponding isoscalar and isovector ones [16]. The comparison among these macroscopic isoscalar transition densities and the RPA-based microscopic one is shown in fig. 2 . The shapes of the three isoscalar transition densities are similar but they are quite different in their magnitude especially at the nuclear surface region which is the region explored by the nuclear reaction considered here. In particular, the one calculated by Harakeh and Dieperink [20], dashed blue curve (HD), are bigger with respect to the RPA one, both inside the nucleus and at the surface. This is not surprising because this transition density has been constructed for a pure isoscalar mode as it is the ISGDR. Indeed for this state the comparison with the microscopic RPA transition density is very good being the two almost undistinguishable [16]. The isoscalar transition density calculated according to the macroscopic pygmy model described in ref. [16], denoted as MPM in the figure with a dot-dashed red line, follows closely the RPA one but it is smaller in the important surface region. All the transition densities have been calculated from the HF ground state density used in the RPA calculations. 
In constructing the formfactor with the double folding procedure we use the M3Y [21] nucleon-nucleon interaction together with the HF ground state density and the transition densities shown in fig. 2. As an example we have chosen the system ${ }^{68} \mathrm{Ni}+{ }^{12} \mathrm{C}$ and their nuclear form factors are shown in fig. 3. The legend indicate which of the three transition densities shown in fig. 2 have been used for the form factor calculations. The three curves are different in all the radial interval; at the surface they differ in magnitude as well as in the slope, the RPA having a slightly larger radial extension. We recall the fact that the excitation processes we are dealing with are mainly active on the surface

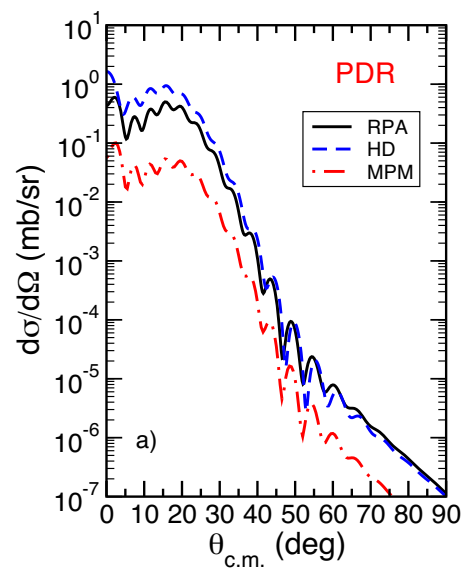

Figure 4: (Color online) Comparison among angular distributions calculated with the form factors of fig.3 (see text). and therefore the most important parts of the form factor are the ones around $8 \mathrm{fm}$ for the system under consideration. In this region the three form factors are quite different and as a consequence one can expect that the corresponding inelastic cross section can be different. Indeed, the almost factor three in the form factor gives rise to an almost factor 9 in the cross section shown in fig. 4 . These were obtained by DWBA calculations for the system ${ }^{68} \mathrm{Ni}+{ }^{12} \mathrm{C}$ at $10 \mathrm{Mev} / \mathrm{u}$ with the DWUCK4 code [22].

\section{Conclusion}

The study of the PDR via isoscalar mode is a well established method [23,24]. The information extracted form the experiment depends on the radial form factors used in the data analysis. Here we have shown that there are strong differences when macroscopic transition densities are used in comparison with the microscopic ones. On the other hand, only the microscopic form factor includes, by construction, the important isospin mixing of the PDR states. Our microscopic form factors have already been successfully employed in the analysis of various experiments performed with ${ }^{17} \mathrm{O}$ [13-15].

\section{References}

[1] N. Paar, D. Vretenar, E. Khan and G. Colò, Rep. Prog. Phys. 70 (2007) 691. 
[2] D. Savran, T. Aumann and A. Zilges, Prog. Part. Nucl.Phys. 70 (2013) 210.

[3] A. Bracco, F. C. L. Crespi and E. G. Lanza, Eur. Phys. J. A 99 (2015)

[4] J. Piekarewicz, Phys. Rev. C 73044325 (2006).

[5] J. Piekarewicz et al., Phys. Rev. C 85041302 (2012).

[6] E. Litvinova et al., Nucl. Phys. 82326 (2009).

[7] P. Adrich et al., Phys. Rev. Lett. 95132501 (2005).

[8] O. Wieland et al., Phys. Rev. Lett. 102092502 (2009).

[9] D. Savran et al., Phys. Rev. Lett. 97172502 (2006).

[10] J. Endres et al., Phys. Rev. C 80034302 (2009).

[11] J. Endres et al., Phys. Rev. Lett. 105212503 (2010).

[12] J. Endres et al., Phys. Rev. C 85064331 (2012).

[13] F. C. L. Crespi et al., Phys. Rev. Lett. 113012501 (2014).

[14] L. Pellegri et al., Phys. Lett. B 738519 (2014).

[15] F. C. L. Crespi et al., Phys. Rev. C 91024323 (2015).

[16] E.G. Lanza, A. Vitturi, M.V. Andrés, Phys. Rev. C 91 (2015) 054607.

[17] G. R. Satchler and W. G. Love, Phys. Rep. 55183 (1979).

[18] N. Van Giai and H. Sagawa, Phys. Lett. B 106379 (1981).

[19] T. J. Deal, Nucl. Phys. A 217210 (1973).

[20] M. N. Harakeh and A. E. L. Dieperink, Phys. Rev. C 232329 (1981).

[21] G. Bertsch et al., Nucl. Phys. A 284399 (1977).

[22] P. D. Kunz, http://spot.colorado.edu/?kunz/DWBA.html

[23] E. G. Lanza, A. Vitturi, M. V. Andrés, F. Catara and D. Gambacurta: Phys. Rev. C 84 (2011) 064602.

[24] E. G. Lanza, A. Vitturi, E. Litvinova and D. Savran: Phys. Rev. C 89 (2014) 041601(R). 